ADOLESCENTS' SLEEP/WAKE PATTERNS AND SCHOOL SCHEDULES:

TOWARDS FLEXIBILITY

\author{
Fernando Mazzilli Louzada ${ }^{1, *}$ \\ Sofia Isabel Ribeiro Pereira ${ }^{2}$ \\ 1 - Human Chronobiology Laboratory, Department of Physiology, Federal University of Paraná, \\ 81531-990 Curitiba, Paraná, Brazil \\ 2 - Psychology Department, CUBRIC, University of Cardiff
}

*Corresponding author flouzada@ufpr.br

(Received 10 June 2018; final version received 14 June 2018)

\title{
ABSTRACT
}

In the literature, two main trends may be identified in the countermeasures associated to adolescents' sleep restriction: sleep education and changes in the school starting times. However, school educational programs have been able to increase knowledge about sleep, which did not necessarily result in behavioral changes. Later school start times have been proposed as a strategy to reduce the adolescents' sleep restriction but there is no consensus about the change. Longitudinal studies in adolescent populations are urgently needed in order to determine the efficacy of interventions aiming at a change in school starting times. Academic, behavioural, health and wellbeing variables could be monitored for an extensive period before and after implementing the new school schedule options, thereby enabling a within-subject experimental design. It is no longer acceptable to impose the 'one size fits all' approach on the school start times.

\section{INTRODUCTION - THE STATE OF THE ART}

Our current understanding of adolescents' sleep-wake patterns is far from negligible. Underlying bioregulatory processes have been described and environmental factors influencing it have been identified (Peixoto et al. 2009; Tarokh et al. 2016; Troxel \& 
Wolfson 2017). Mechanisms associated to the delay in the adolescents' daily timing of the sleep-wake cycle have been proposed (Andrade et al. 1993; Carskadon et al. 1993; Hummer \& Lee 2016). It seems that pubertal changes in the homeostatic drive to sleep would permit adolescents to stay up later in the evening (Jenni et al. 2005; Hummer \& Lee 2016).

In parallel, the importance of sleep for health, cognition and overall well-being is extensively documented in the literature (McCoy \& Strecker 2011; Itani et al. 2016; Krueger et al. 2016). Neurocognitive effects of sleep restriction have also been demonstrated (Lowe et al. 2017). Besides, sleep has not only an impact upon the brain's readiness to learn, but it also contributes to memory consolidation after learning (Benson \& Feinberg 1977; Diekelmann \& Born 2010).

However, little progress has been made in increasing the number of adolescents who are obtaining adequate sleep (Troxel \& Wolfson 2017). Insufficient sleep duration in adolescents has been described in many studies, in different countries and settings (Shin et al. 2003; Loessl et al. 2008; Chen et al. 2014; Merdad et al. 2014). Actually, Keyes et al. reported a decline in sleep duration among US adolescents in the last two decades (Keyes et al. 2015).

In summary, the majority of the studies show that a significant percentage of teens sleep less than the National Sleep Foundation recommendations ranging from 8 to 10 hours of sleep per day (Hirshkowitz et al. 2015).

In the literature, two main strands can be identified in the countermeasures associated to adolescents' sleep restriction: sleep education and changes in the school starting times (SST). In this text, we first intend to go deeper in each one and then discuss proposals for new research and interventions in the field.

\section{SLEEP EDUCATIONAL PROGRAMS}

Studies evaluating the effects of sleep educational programs in adolescents are scarce in the literature. In 2012, Blunden et al. published a review in which they reported to find only 8 studies and 4 pilot studies in abstract form (Blunden et al. 2012). In subsequent years, the number of studies has not increased significantly. Four years later, Gruber found 15 studies in his review (Gruber 2016).

Cortesi et al. evaluated the effect of a sleep educational program upon the knowledge about sleep on a group of Italian adolescents. The program consisted of a 2-hour lesson conducted by a sleep professional. The authors concluded that sleep educational programs are effective in improving information about sleep (Cortesi et al. 2004). 
A school-based intervention for adolescent sleep problems, based on a cognitive-behavior therapy framework, was evaluated. The intervention consisted in four 50-min classes across four weeks. Adolescents increased sleep knowledge but no significant effects on target sleep variables were observed (Moseley, Clin, and Gradisar, 2009).

Beijamini and Louzada evaluated the effect of an educational sleep program on adolescents' sleep patterns and daytime sleepiness. The results did not show any effect of the program on the adolescents' sleep/wake patterns, subjective sleepiness levels or psychomotor performance (Beijamini \& Louzada 2012).

A decrease in sleep irregularity was observed in adolescents after a sleep education program which consisted of five 50-minute classes to discuss the physiological and behavioral processes of sleep and healthy lifestyle (Sousa et al. 2013). No changes in other sleep parameters were observed.

More recently, in 2017, the Sleep Medicine Reviews Journal published a guest editorial with the title: "What more can we learn about sleep education programs in young people?" In the editorial, Blunden has brought again the discussion of the main challenge in the field, how to enhance the translation of knowledge into actual sleep behavior change taking into account that long-term maintenance of the changes has not been observed in the studies (Blunden 2017). Motivation to change is another important factor to achieve the desirable outcomes (Cain et al. 2011). Another concern related to sleep educational programs is if the changes observed in sleep patterns are of clinical significance (Blunden, 2017).

In summary, school educational programs have been able to increase knowledge about sleep, which did not necessarily result in behavioral changes. Researchers need to increase collaboration in order to standardize messages, content and outcomes measured (Blunden, 2017).

\section{SCHOOL SCHEDULES CHANGES}

Early school start times may exacerbate the already challenging task for adolescents of obtaining the needed amount of night sleep during school days (Appleman et al. 2015). The effect of early school starting times can be clearly observed when afternoon versus morning school shifts are compared. Changing from the afternoon to the morning shift, a common practice among Brazilian schools, is associated to a decrease on sleep duration (Mello et al., 2001; Brandalize et al. 2011). 
Later school start times have been proposed as a strategy to reduce the adolescents' sleep restriction (Lewin et al. 2017). On the other hand, in 2016, Minges and Redeker, in a systematic review, claim that there has been no systematic synthesis of the evidence on the effects of this practice. According to them, there is a need for more rigorous randomized study designs, including objective sleep measures and consistent health and academic performance measures (Minges \& Redeker 2016). In line with this view, Marx et al. have conducted a systematic review and concluded that, with the available data, is not possible to determine the effects of later SST with confidence. The authors also pointed the need for higher quality primary studies (Marx et al. 2017).

In spite of these controversies, in recent years a significant number of studies related to SST changes have been published. A considerable number of studies have shown that later school start times (SST) are associated with positive student outcomes: improvements in academic performance, mental and physical health and public safety (Hafner et al. 2017). Students with SST before 7:45 A.M. can be exposed to an increased risk of sleep duration curtailment, poorer academic performance and academic effort (Lewin et al. 2017).

Owens et al. evaluated the impact of a 30-minute delay in SST on adolescents' sleep, mood, and behavior and observed an 18 minute advance in bedtime, a reduction in the percentage of students reporting less than 7 hours of sleep and an increase in those reporting more than 8 hours of sleep. Additionally, students reported reduction on fatigue and depressed mood (Owens et al. 2017).

The relationships between high school starting times, adolescent sleep duration, mental health and substance use related issues and behaviors were examined from the Teen Sleep Habits Survey in the United States. It was found that later starting times were significantly associated with greater sleep duration and greater sleep duration was associated with fewer reports of various mental health and substance use related issues and behaviors (Wahlstrom et al. 2017).

Patte et al. investigated school SST in relation to sleep duration and adherence to guidelines among Canadian youth. The sample included 35,821 grade 9-12 students at 78 secondary schools in Ontario and Alberta, Canada, from COMPASS study. For each hour delay in SST, students reported an average of almost 7 minutes longer sleep and had 1.17 higher odds of meeting the recommendations (Patte et al. 2017).

The effects of SST on sleep duration of students were examined in a metaanalysis which included five longitudinal studies and 15 cross-sectional comparison group studies. Results indicated that later SSTs were associated with longer sleep 
durations. In 7 of the examined studies later SSTs were associated with less daytime sleepiness (Bowers \& Moyer 2017).

A quasi-experimental study in United States public schools was conducted using cross-sectional surveys before and after changes of school starting times to investigate the impact on adolescents' sleep. They investigated a 50-minute delay (7:20 to $8: 10 \mathrm{AM})$ in starting time for high schools and secondary schools and a 30 minute advance (8:00 to 7:30 AM) for middle schools. Students with a 50-minute delay slept 30.1 minutes longer on school nights and had less daytime sleepiness, whereas students with a 30-minute advance slept 14.8 minutes less and had more daytime sleepiness (Owens et al. 2017).

Sleep patterns of 413 North American high schools students submitted to different starting times were compared. Teens with the earliest SST (7:00-7:29 AM) obtained 46 minutes less time in bed on average compared with teens with school start times at 8:30 AM or later (Nahmod et al. 2017).

McKeever and Clark investigated changes in high school graduation rates and attendance rates 2 years after a delay in SST in more than 30,000 students attending 29 high schools located throughout seven US different states and found that attendance rates and graduation rates significantly improved in schools with delayed start times of 8:30AM or later (McKeever \& Clark 2017).

Sustainable benefits after a delay in SST, from 7:30 to 8:15, was observed in female high school students in Singapore. After 9 months, the short-term increase in time in bed was sustained, and total sleep time increased by 10 min relative to baseline (Lo et al. 2018).

More recently, a prioritization of policy initiatives to implement healthy SST for younger adolescents has been discussed. In 2014, The American Academy of Pediatrics (AAP) released a policy statement supporting the efforts of US school districts to change SST. The Center for Disease Control and Prevention (CDC) has also published material with similar concerns. Barnes et al. (2016) published a call to action for the implementation of AAP/CDC recommendations across the United States. In addition, Dunietz et al. pointed out the importance of parental education about sleep needs in order to increase their support for later SST (Dunietz et al. 2017). In 2017, Sleep Health, Journal of National Sleep Foundation, published a special issue on school starting times. According to the editors, "Later school starting times and the implications for adolescent sleep represent an important and rare example of the intersection between sleep science and education policy" (Troxel \& Wolfson 2017). The editors pointed out that only one modifiable policy level countermeasure had been identified: early school starting times. 
However, there is no consensus about the change. One criticism is related to methodological flaws in the studies previously mentioned. Most of the studies are cross-sectional and thus cannot demonstrate that early SST cause negative outcomes. This argument has been rebutted by the fact that the reverse direction of causality is implausible (behavior problems causing school start times) (Keller et al. 2017). There are very few experimental studies of the effects of school start times on sleep or daytime functioning for any age. Actually, it would be almost impossible to conduct a randomized, double-blind trial to test the effects of delayed SST. Another argument against the changes in SST is that delaying SST will affect the transportation system. Hafner et al. used a novel macroeconomic modeling approach to estimate changes in the economic performance of 47 US states following a delayed school start times and concluded that it is a cost effective strategy (Hafner, Stepanek, and Troxel, 2017).

\section{PERSPECTIVES - TOWARDS FLEXIBILITY}

Longitudinal studies in adolescent populations are urgently needed in order to determine the efficacy of interventions aiming at a change in SST. Academic, behavioural, health and wellbeing variables could be monitored for an extensive period before and after implementing the new school schedule options, thereby enabling a within-subject experimental design. Given the significant variation in individual circadian preferences, it is of paramount importance to contemplate both morning and evening-type needs by offering the possibility to choose between earlier and later school starting (and finishing) times. This format is commonly adopted in universities, where a large class is subdivided into smaller groups taking turns attending the same courses in alternating times. This approach is particularly helpful when the students are engaged in group and/or practice activities, usually in a lab (chemistry, biology, physics, informatics, robotics, foreign languages, etc.), since it provides the opportunity for closer one-on-one interactions between students and between the students and the professor. Nowadays there is a wide array of technological devices, softwares and systems especially designed for educational settings that could be implemented to facilitate the transition into the flexible SST proposal. Given our current understanding of the importance of sleep in learning, memory, the immune system and metabolism (reviewed in Rasch \& Born, 2013), it is no longer acceptable to impose the 'one size fits all' approach on the school start times. Even in the corporate world many companies have adopted flexible working hours, since it has been shown to increase productivity, self-engagement and motivation and to decrease absenteeism and turnover (Shepard III et al. 1996). In the United Kingdom, for example, all employees have the legal right 
to request flexible working - not just parents and carers (https://www.gov.uk/flexibleworking), so why should we continue to deprive our adolescents and their developing brains and bodies of this opportunity? 


\section{REFERENCES}

Andrade MM, Benedito-Silva a a, Domenice S, Arnhold IJ, Menna-Barreto L. 1993. Sleep characteristics of adolescents: a longitudinal study. J Adolesc Health. 14:401-406.

Appleman ER, Gilbert KS, Au R. 2015. School start time changes and sleep patterns in elementary school students. Sleep Health. 1:109-114.

Beijamini F, Louzada FM. 2012. Are educational interventions able to prevent excessive daytime sleepiness in adolescents? Biol Rhythm Res. 43:603-613.

Benson K, Feinberg I. 1977. The beneficaial effect of sleep in an extended Jenkins and Dallenbach paradigm. Psychophysiology. 14:375-384.

Blunden SL. 2017. What more can we learn about sleep education programs in young people? Sleep Med Rev. 36:1-2.

Blunden SL, Chapman J, Rigney G a. 2012. Are sleep education programs successful? The case for improved and consistent research efforts. Sleep Med Rev. 16:355-370.

Bowers JM, Moyer A. 2017. Effects of school start time on students' sleep duration, daytime sleepiness, and attendance: a meta-analysis. Sleep Health. 3:423-431.

Brandalize M, Pereira ÉF, Leite N, Filho GL, Louzada FM. 2011. Effect of morning school schedule on sleep and anthropometric variables in adolescents: A follow-up study. Chronobiol Int. 28:779-785.

Cain N, Gradisar M, Moseley L. 2011. A motivational school-based intervention for adolescent sleep problems. Sleep Med. 12:246-251.

Carskadon M a., Vieira C, Acebo C. 1993. Association between puberty and delayed phase preference. Sleep. 16:258-262.

Chen T, Wu Z, Shen Z, Zhang J, Shen X, Li S. 2014. Sleep duration in Chinese adolescents: Biological, environmental, and behavioral predictors. Sleep Med. 15:1345-1353.

Cortesi F, Giannotti F, Sebastiani T, Bruni O, Ottaviano S. 2004. Knowledge of sleep in Italian high school students: Pilot-test of a school-based sleep educational program. J Adolesc Heal. 34:344-351.

Diekelmann S, Born J. 2010. The memory function of sleep. Nat Rev Neurosci. 11:114-126.

Dunietz GL, Matos-Moreno A, Singer DC, Davis MM, OBrien LM, Chervin RD. 2017. Later school start times: What informs parent support or opposition? J Clin Sleep Med. 13:889-897.

Gruber R. 2016. School-Based Sleep Education Programs: A Knowledge-To-Action Perspective Regarding Barriers, Proposed Solutions, and Future Directions. Sleep Med Rev.1:16.

Hafner M, Stepanek M, Troxel WM. 2017. The economic implications of later school start times in the United States. Sleep Health. 3:451-457.

Hirshkowitz M, Whiton K, Albert SM, Alessi C, Bruni O, DonCarlos L, Hazen N, Herman J, Katz ES, Kheirandish-Gozal L, et al. 2015. National sleep foundation's sleep time duration recommendations: Methodology and results summary. Sleep Health. 1:40-43.

Hummer DL, Lee TM. 2016. Daily Timing of the Adolescent Sleep Phase: Insights from a CrossSpecies Comparison. Neurosci Biobehav Rev. 70:171-181.

Itani O, Jike M, Watanabe N, Kaneita Y. 2017. Short sleep duration and health outcomes: A systematic review, meta-analysis and meta-regression. Sleep Med. 32:246-256 
Jenni OG, Achermann P, Carskadon M a. 2005. Homeostatic sleep regulation in adolescents. Sleep. 28:1446-1454.

Keller PS, Gilbert LR, Haak E a., Bi S, Smith O a. 2017. Earlier school start times are associated with higher rates of behavioral problems in elementary schools. Sleep Health. 3:113-118.

Keyes KM, Maslowsky J, Hamilton a., Schulenberg J. 2015. The Great Sleep Recession: Changes in Sleep Duration Among US Adolescents, 1991-2012. Pediatrics. 135:460-468.

Krueger JM, Frank MG, Wisor JP, Roy S. 2016. Sleep function: Toward elucidating an enigma. Sleep Med Rev. 28:42-50.

Lewin DS, Wang G, Chen YI, Skora E, Hoehn J, Baylor A, Wang J. 2017. Variable School Start Times and Middle School Student's Sleep Health and Academic Performance. J Adolesc Heal. 61:205-211.

Lo JC, Lee SM, Lee XK, Sasmita K, Chee NIYN, Tandi J, Cher WS, Gooley JJ, Chee MWL. 2018. Sustained benefits of delaying school start time on adolescent sleep and well-being. Sleep.:1-8.

Loessl B, Valerius G, Kopasz M, Hornyak M, Riemann D, Voderholzer U. 2008. Are adolescents chronically sleep-deprived? An investigation of sleep habits of adolescents in the Southwest of Germany. Child Care Health Dev. 34:549-556.

Lowe CJ, Safati A, Hall P a. 2017. The neurocognitive consequences of sleep restriction: A meta-analytic review. Neurosci Biobehav Rev. 80:586-604.

Marx R, Tanner-Smith EE, Davison CM, Ufholz L-A, Freeman J, Shankar R, Newton L, Brown RS, Parpia AS, Cozma I, Hendrikx S. 2017. Later school start times for supporting the education, health and well-being of high school students (Protocol) Later school start times for supporting the education, health and well-being of high school students. Cochrane Database Syst Rev.

McCoy JG, Strecker RE. 2011. The cognitive cost of sleep lost. Neurobiol Learn Mem. 96:564582.

McKeever PM, Clark L. 2017. Delayed high school start times later than 8:30 AM and impact on graduation rates and attendance rates. Sleep Health. 3:119-125.

Mello, L., Louzada, F.\&Menna-Barreto, L, 2001. Effects of School-schedules transition on sleep/wake cycle of Brazilian students. Sleep and Hypnosis. 3:3,106-111.

Merdad R a., Merdad L a., Nassif R a., El-Derwi D, Wali SO. 2014. Sleep habits in adolescents of Saudi Arabia; distinct patterns and extreme sleep schedules. Sleep Med. 15:1370-1378.

Minges KE, Redeker NS. 2016. Delayed school start times and adolescent sleep: A systematic review of the experimental evidence. Sleep Med Rev. 28:82-91.

Moseley L, Clin MP, Gradisar M. 2009. Evaluation of a School-Based Intervention for Adolescent Sleep Problems. 32(3):334-41.

Nahmod NG, Lee S, Buxton OM, Chang AM, Hale L. 2017. High school start times after 8:30 $\mathrm{AM}$ are associated with later wake times and longer time in bed among teens in a national urban cohort study. Sleep Health. 3:444-450.

Owens J a., Dearth-Wesley T, Herman AN, Oakes JM, Whitaker RC. 2017. A quasiexperimental study of the impact of school start time changes on adolescent sleep. Sleep Health. 3:437-443.

Patte K a., Cole AG, Qian W, Leatherdale ST. 2017. Youth sleep durations and school start times: a cross-sectional analysis of the COMPASS study. Sleep Health. 3:432-436. 
Peixoto CAT, da Silva AGT, Carskadon M a, Louzada FM. 2009. Adolescents living in homes without electric lighting have earlier sleep times. Behav Sleep Med. 7:73-80.

Rasch, B.\& Born,J., 2013. About sleep's role in memory.Physiol.Rev. 93(2):681-766.

Shepard III EM, Clifton TJ, Kruse D. 1996. Flexible work hours and productivity: Some evidence from the pharmaceutical industry. Ind Relat (Berkeley). 35:123-139.

Shin C, Kim J, Lee S, Ahn Y, Joo S. 2003. Sleep habits, excessive daytime sleepiness and school performance in high school students. Psychiatry Clin Neurosci. 57:451-453.

Sousa IC, Souza JC, Louzada FM, Azevedo CVM. 2013. Changes in sleep habits and knowledge after an educational sleep program in 12th grade students. Sleep Biol Rhythms. $11: 144-153$.

Tarokh L, Saletin JM, Carskadon M a. 2016. Sleep in adolescence: Physiology, cognition and mental health. Neurosci Biobehav Rev. 70:182-188.

Troxel WM, Wolfson AR. 2017. The intersection between sleep science and policy: introduction to the special issue on school start times. Sleep Health. 3:419-422.

Wahlstrom KL, Berger AT, Widome R. 2017. Relationships between school start time, sleep duration, and adolescent behaviors. Sleep Health. 3:216-221. 